\title{
The CORES Project
}

\author{
P.A. Cudd ${ }^{+}$, M. Freeman ${ }^{+}$, R.B. Yates ${ }^{+}$, A. J. Wilson ${ }^{+}$, M.P. Cooke ${ }^{+}$, M.S. Hawley* \\ + University of Sheffield, UK * Barnsley District General Hospital, UK
}

\begin{abstract}
Discussion paper of a proposed project that is the initial phase of a far reaching research programme. The CORES project aims to investigate the development of a speech-only-input based Human Computer Interface (HCI) which will lead to suitably skilled disabled users being able to operate any commonly available commercial personal computer or workstation. The long term aim is to generalise this to a portable device with an adaptable HCI (both in hardware and software terms) suitable for disabled and able-bodied people alike.
\end{abstract}

\section{Introduction}

The UK lags North America and Scandinavia in establishing multi-disciplinary research and development centres in the fields of Rehabilitation and HumanMachine Interfacing. There is a direct correlation between the higher levels of this type of research and the degree of productive employment and social integration of disabled people in these geographical regions. It is of note that the industrial sector has also recognised that applications of technology for disability has mass market applications among the general population ${ }^{1}$.

The technology required to achieve rehabilitation becomes more complex when those with severe physical disabilities are considered ${ }^{2,3,4}$. For such individuals a very limited number of controllable movements are available to operate devices which replace the motor and communication functions. Current assistive devices clearly benefit disabled people, but they do not provide communication or motor function at the speed or response that is required for the activities of daily living.

Electronic and computer technology has a major role to play in the process of optimising the control of devices to substitute impaired function, both in its ability to control flexible displays for presentation of options and control information to the user, and, to process historical sequences of actions to predict those which are most likely to be selected next. The potential of Automatic Speech Recognition to enhance the HMI is great, but many issues have still not been fully investigated, e.g. safety for control applications; automatic recognition for impaired speech; and tailored Human-Computer Interfacing, in terms of both software and hardware ${ }^{2}$.

The CORES project aims to go beyond current investigations and initiate a five to ten year research programme. The name 'CORES' is derived from 'COmmunication and Rehabilitation Engineering at Sheffield'. The project will contribute to the long term goal to develop a Universal Human-Machine Interface (UHMI), i.e. a portable device that would facilitate the operation of any type of computer or industrial/domestic electronic control system by either able-bodied or disabled individuals. Thus the concept goes beyond existing research $2,3,4$.

Many disabled people have both limited and uncontrolled limb movements while still having some speech, but that may also be severely impaired. Nonetheless, speech offers a useful alternative to other input modalities. Thus the CORES project will aim to result in a design framework for the UHMI and a demonstrator with impaired-speech input that will facilitate the use of commercial computers. 
For more than a decade the cognitive processes associated with human tasks have been analysed to improve the effectiveness of the HCI. Through fundamental research, this branch of systems development has now established a well founded theoretical framework in which computer interfaces can be designed. Unfortunately, little of this work has either been applied to the development of generic electronic systems for disabled users or to include consideration of the optimum hardware. So currently commercially available state of the art HCIs such as Microsoft Windows ${ }^{\text {TM }}$ and $\mathrm{X}$-Windows ${ }^{\mathrm{Tm}}$ require an input modality transducer for non-standard input devices. Interaction (i.e. control of) computers by disabled users is an area that has received some attention. However, the HCI aspects of the existing systems to be used, i.e. the standard hardware input devices and the operational characteristics of the software application, have been accepted as a starting specification. So, problems, or at least awkwardness of use, appear because the input device and the feedback needs of the user were not part of the original HCI. The CORES project demonstrator system aims to provide a tailored interface for the chosen input modality (speech) which maximises independence of the applicationuser interface. Implementation of the demonstrator based on user- and applicationadaptable state of the art hardware gives the CORES project a significant edge over previous approaches to HMI for the disabled. The work of CORES therefore points to the possibility in the future of bespoke user interfaces for everyone; in the more immediate future this may only be justifiable for specialist applications.

In industrial and business applications increasing amounts of computer system processing power is devoted to the user interface, viz. Graphical User Interfaces, multi-media, speech recognition and virtual reality. Current hardware implementations are not tailored to these input/output $(\mathrm{I} / \mathrm{O})$ tasks, and consequently the implementations of even well- researched and designed HMIs are frequently slow. When such interfaces are considered for use by the disabled community, they are not sufficiently adaptable to cope with the extra-ordinary needs. These factors have led to the recognition of the need for a new approach in designing a Human Machine Interface (HMI). Future implementations of HMIs should be portable and be conceived as a quite separate I/O device to the 'application tool'. The latter 'application tool' could be a computer or an industrial/domestic electronic system. Of particular interest, therefore, is a framework for the development and evaluation of a modular and integrated Universal Human Machine Interface (UHMI). This would include software, operating system and hardware, and be modular in terms of its $1 / O$ capabilities, these modules being tailorable to the appropriate requirements of the user and the attached 'application tool'. Such a framework would bring together the host application (e.g. word processor) with components of communications technology (e.g. speech recogniser) and the user-application interaction.

\section{Work to be carried out}

The first task is to create a requirements specification for the framework, using information from related work such as in the area of telecoms 5 . Specification of 
hardware for the demonstrator will also be initiated. Next, the framework will be developed using available communications technology resulting in a demonstrator. Preliminary evaluations would be carried out and reported. Lastly, investigation of more specific day to day and long term needs of disabled individuals would be addressed, e.g. casual communication, and, facilitating education and employment. It is difficult at to give more precise details on the work to be carried out until the requirements specification has been achieved, but we expect to consider the following themes taking full advantage of existing HMI tools and experience:

- Existing speech recognisers and impaired speech

- The parameters of impaired speech

- Gesture recognition based on recent advances in machine vision

- Input systems based on movement analysis

- Actuator feedback and cognitive issues

- Use of parallel input and output modalities

- Adaptive HMI

- Commonality with applications using processing intensive user interfacing.

It is envisaged that the user will effect control through supplying input to a device which 'connects' this input to the computer system in such a way that manipulation of the processes on the computer is possible. It is planned that computer platform independence will be achieved through use of those input/output connections that change little with time, e.g. mouse, keyboard and display ports. It is intended to use recent advances in 2D object recognition and ASICs to achieve automatic recognition of screen activity.

\section{Conclusions}

Commonality with other processing intensive user-interfacing applications, such as Virtual Reality, means that the future holds a greater opportunity to achieve more powerful, bespoke and hence more appropriate user interfaces for disabled individuals. The complexity of augmenting or assisting impaired functions will, by necessity, mean that the approach to the technology required to realise user-friendly and use-efficient systems be both complex and innovative. Development of bespoke user interfaces in the Rehabilitation field has useful implications and indeed may lead the way for the general Human-computer/machine-interfacing of the future.

Acknowledgement : The authors are indebited to Dr. P. Courtney.

\section{References}

1. The Apple Macintosh and Disability Access Syllabus, 1994.

2. Hawley, M.S., Cudd, P.A. and Cherry, A.D. : 'Implementation of a PC-based integrated control system for children', Med. Eng. Phys., May 1994, Vl. 16, 237-42.

3. Buhler, C. : 'Uniform user interface for communication and control', Proceedings ECART2, Stockholm, May 1993, pp22.3.

4. van Woerden, J.A. : 'M3S A General Purpose Interface for the Rehabilitation Environment', Proceedings ECART2, Stockholm, May 1993, pp22.1.

5. von Tetzchner, S. (Editor) : 'Issues in Telecommunication and Disability', CEC, EUR 13845 EN, ISBN 92-826-3128-1, 1991 